\title{
AN INJECTIVE FAR-FIELD PATTERN OPERATOR AND INVERSE SCATTERING PROBLEM IN A FINITE DEPTH OCEAN
}

\author{
by YONGZHI XU*
}

(Received 28th August 1989)

\begin{abstract}
The inverse scattering problem for acoustic waves in shallow oceans are different from that in the spaces of $\mathbf{R}^{2}$ and $\mathbf{R}^{3}$ in the way that the "propagating" far-field pattern can only carry the information from the $N+1$ propagating modes. This loss of information leads to the fact that the far-field pattern operator is not injective. In this paper, we will present some properties of the far-field pattern operator and use this information to construct an injective far-field pattern operator in a suitable subspace of $L^{2}(\partial \Omega)$. Based on this construction an optimal scheme for solving the inverse scattering problem is presented using the minimizing Tikhonov functional.
\end{abstract}

1980 Mathematics subject classification (1985 Revision): 35R30, 76Q05.

\section{Introduction}

The inverse scattering problem for acoustic waves, which consists in recovering the shape of a scatterer from the far-field pattern of the scattered field, forms the basis of a wide variety of areas in the engineering sciences such as remote sensing, nondestructive testing and imaging etc., and for this reason has been the object of study by scientists in a number of diverse disciplines. Rapid progress in this field has been made since the early seventies, and a survey of these results can be found in the papers by Colton [4] and Sleeman [12]. However, nearly all intensive efforts in this field are devoted to the cases of $\mathbf{R}^{2}$ and $\mathbf{R}^{3}$. It has been noticed that in some situations, for instance in a finite depth ocean, the remote sensing and imaging problems will lead to an inverse scattering problem in a special space instead of $\mathbf{R}^{2}$ and $\mathbf{R}^{3}$. In the homogeneous finite depth ocean, Gilbert and Xu [8] showed that the "propagating" far-field pattern can only carry the information from the $N+1$ propagating modes; here $N$ is the largest integer less than $(2 k h-\pi) / 2 \pi$. This loss of information makes this problem different from that in whole space case in the way that the far-field pattern operator is not injective.

Before we can describe this non-injective property of the far-field pattern more precisely, we need to give a formulation of the corresponding direct problem, that is of the exterior boundary value problem for the time harmonic acoustic scattering by a soft object.

*This work is supported in part by Sea Grant NA86AA-D-SG040. 
Let $\mathbf{R}_{b}^{3}=\left\{(\mathbf{x}, z) ; \mathbf{x}=\left(x_{1}, x_{2}\right) \in \mathbf{R}^{2}, 0 \leqq z \leqq h\right\}$ be a region corresponding to the finite depth ocean, where $h$ is the ocean depth. Let $\Omega$ be an object imbedded in $\mathbf{R}_{b}^{3}$, which is a bounded, convex domain with $C^{2}$ boundary $\partial \Omega$ having an outward unit normal $v$. If the object has a sound soft boundary $\partial \Omega$, an incoming wave $u^{i}$, which is incident on $\partial \Omega$, will be scattered to produce a propagating wave $u^{s}$ as well as its far-field pattern. This problem can be formulated as a Dirichlet boundary value problem for the scatttering of time-harmonic acoustic waves in $\Omega_{e}:=\mathbf{R}_{b}^{3} \backslash \Omega$, namely to find a solution $u \in C^{2}\left(\mathbf{R}_{b}^{3} \backslash \vec{\Omega}\right) \cap$ $\mathbf{C}\left(\mathbf{R}_{b}{ }^{3} \backslash \Omega\right)$ to the Helmholtz equation

$$
\Delta_{3} u+k^{2} u=0, \quad \text { in } \quad \mathbf{R}_{b}^{3} \backslash \bar{\Omega}
$$

such that $u$ satisfies the boundary conditions

$$
\begin{gathered}
u=0, \quad \text { as } \quad z=0 \\
\frac{\partial u}{\partial z}=0, \quad \text { as } \quad z=h \\
u=0, \quad \text { on } \quad \partial \Omega .
\end{gathered}
$$

Here $k \neq(2 n+1) \pi / 2 h, h=0,1, \ldots, \infty$ is a positive constant known as the wave number, and $u=u^{i}+u^{s}$, where $u^{i}$ and $u^{s}$ are the incident (entire) wave and the scattered wave respectively. The scattered wave has the modal representation

$$
u^{s}=\sum_{n=0}^{\infty} \phi_{n}(z) u_{n}^{s}(\mathbf{x})
$$

where

$$
\begin{aligned}
& \phi_{n}(z)=\sin \left[k\left(1-a_{n}^{2}\right)^{1 / 2} z\right], \\
& a_{n}=\left[1-\frac{(2 n+1)^{2} \pi^{2}}{4 k^{2} h^{2}}\right]^{1 / 2},
\end{aligned}
$$

and the $n$th mode of $u^{s}, u_{n}^{s}(\mathbf{x})$, satisfies the radiating condition

$$
\lim _{r \rightarrow \infty} r^{1 / 2}\left(\frac{\partial u_{n}^{s}}{\partial r}-i k a_{n} u_{n}^{s}\right)=0, \quad r=|\mathbf{x}|, \quad n=0,1, \ldots, \infty
$$

This problem is uniquely solvable [14]. Let $G(z, \zeta,|\mathbf{x}-\xi|)$ be the Green's function in $\mathbf{R}_{b}^{3}$ satisfying boundary condition (1.2) and (1.3), then the scattered wave $u^{s}$ can be represented as 


$$
u^{s}(\mathbf{x}, z)=\int_{\partial \Omega}\left(u \frac{\partial G}{\partial v}-G \frac{\partial u}{\partial v}\right) d \sigma, \quad(\mathbf{x}, z) \in \Omega_{e}
$$

and has the asymptotic expansion

$$
u^{s}(\mathbf{x}, z)=\frac{i}{2 h} e^{-i \pi / 4} \sum_{n=0}^{N}\left(\frac{2}{\pi k a_{n} r}\right)^{1 / 2} e^{i k a_{n} r} f_{n}(\theta, z, k)+O\left(\frac{1}{r^{3 / 2}}\right)
$$

where we denote $(\mathbf{x}, z)$ in cylindrical coordinates by $(r, \theta, z)$, and

$$
\begin{gathered}
f_{n}(\theta, z, k)=-\phi_{n}(z) \int_{\partial \Omega} \frac{\partial u(\xi, \zeta)}{\partial v_{\xi}}\left(e^{-i k a_{n} \hat{\mathbf{x}} \cdot \xi} \phi_{n}(\zeta)\right) d \sigma_{\xi}, \\
\hat{\mathbf{x}}=(\cos \theta, \sin \theta), \quad \text { and } \quad N=\left[\frac{2 k h-1}{2 \pi}\right] .
\end{gathered}
$$

Let us denote

$$
V^{N}:=L^{2}[0,2 \pi] \times \operatorname{span}\left\{\phi_{0}, \phi_{1}, \ldots, \phi_{N}\right\}
$$

We then call the function $f(\theta, z, k):=\sum_{n=0}^{N} f_{n}(\theta, z, k) \in V^{N}$ the representation of the propagating far-field pattern of the scattered wave. The operator $F: L^{2}(\partial \Omega) \rightarrow V^{N}$ defined by

$$
\begin{gathered}
(F g)(\theta, z, k):=-\sum_{n=0}^{N} \phi_{n}(z) \int_{\partial \Omega} g(\xi, \zeta)\left(e^{-i k a_{n} \dot{x} \cdot \xi} \phi_{n}(\zeta)\right) d \sigma_{\xi}, \\
\hat{\mathbf{x}}=(\cos \theta, \sin \theta), \quad 0 \leqq \theta \leqq 2 \pi, 0 \leqq z \leqq h .
\end{gathered}
$$

is called a far-field pattern operator (cf. [9]). Unlike the whole space case in which by choosing $\partial \Omega$ properly, from $F \phi=0$ it follows that $\phi=0$ (cf. $[7,10]$ ), here the null space of $F, N(F)$, is not necessarily empty even if $k$ is not an eigenvalue of interior Dirichlet problem on $\Omega$. A particular example of this occurs for $0<k<\pi / 2 h$; then $N=-1$ and for any incoming waves the far-field pattern is identically zero. Even in the case of sufficiently large $k, F \phi=0$ only means that the $N+1$ propagating modes are identically zero. Therefore, the far-field pattern operator $F$ is not an injection over the Hilbert space $L^{2}(\partial \Omega)$.

The inverse scattering problem we wish to consider is as follows: given the far-field pattern $f(\hat{\mathbf{x}}, z, k)$ for one or several incoming (entire) waves, find the shape of the scattering object $\Omega$. In order to solve this problem, we need to find some kind of inverse 
operator of $F$. Therefore, it is important to find out under what kind of restriction $F$ becomes an injection.

In Sections 2 and 3, we will present some properties of the far-field pattern operator and use this information to construct an injective far-field pattern operator in a suitable subspace of $L^{2}(\partial \Omega)$. Based on this construction an optimal scheme for solving the inverse scattering problem is presented using the minimizing Tikhonov functional.

\section{Injective theorem of far-field pattern operator}

In view of $[14,9]$, we can represent the scattered wave $u^{s}$ in the form of combined single and double layer potential:

$$
u^{s}(\mathbf{x}, z)=\int_{\partial \Omega}\left(\frac{\partial}{\partial v_{\xi}}+\lambda\right) G(z, \zeta,|\mathbf{x}-\xi|) g(\xi, \zeta) d \sigma_{\xi}
$$

where $\operatorname{Im} \lambda>0$ and $g(\xi, \zeta)$ satisfies

$$
g+(K+\lambda S) g=-2 u^{i}
$$

Here,

$$
\begin{gathered}
K g:=2 \int_{\partial \Omega} \frac{\partial G}{\partial \nu_{\xi}} g d \sigma, \\
S g:=2 \int_{\partial \Omega} G g d \sigma .
\end{gathered}
$$

$(I+K+\lambda S)$ is invertible for any $k>0, k \neq(2 n+1) \pi / 2 h, n=0,1, \ldots, \infty$, and its inverse is a bounded linear operator in $L^{2}(\partial \Omega)$, denoted by $(I+K+\lambda S)^{-1}$.

For $r=|\mathbf{x}|>|\xi|=: r^{\prime}$, we can expand $G(z, \zeta,|\mathbf{x}-\xi|)$ in the form of a normal mode representation

$$
\begin{gathered}
G(z, \zeta,|\mathbf{x}-\xi|)=\frac{i}{4} \sum_{n=0}^{\infty} \sum_{m=0}^{\infty} \frac{\varepsilon_{m} \phi_{n}(z) \phi_{n}(\zeta)}{\left\|\phi_{n}\right\|^{2}} H_{m}^{(1)}\left(k a_{n} r\right) J_{m}\left(k a_{n} r^{\prime}\right) \\
{\left[\cos (m \theta) \cos \left(m \theta^{\prime}\right)+\sin (m \theta) \sin \left(m \theta^{\prime}\right)\right]}
\end{gathered}
$$

In view of the asymptotic behavior of $H_{m}^{(1)}\left(k a_{n} r\right)$, we can conclude that $u^{s}$ has an asymptotic expression

$$
u^{s}(\mathbf{x}, z)=\frac{i}{2 h} e^{-i \pi / 4} \sum_{n=0}^{\infty}\left(\frac{2}{\pi k a_{n} r}\right)^{1 / 2} e^{i k a_{n} r} \phi_{n}(z)
$$




$$
\left[\sum_{m=0}^{\infty} \varepsilon_{m} \int_{\partial \Omega}\left(\frac{\partial}{\partial v}+\lambda\right) \phi_{n}(\zeta) J_{m}\left(k a_{n} r^{\prime}\right) \cos m\left(\theta-\theta^{\prime}\right) d \sigma\right]+O\left(\frac{1}{r^{3 / 2}}\right)
$$

where $\varepsilon_{0}=1, \varepsilon_{m}=2$ for $m \geqq 1$.

Here a natural way to define the far-field operator is to define $F: L^{2}(\partial \Omega) \rightarrow V^{N}$ by

$$
(F g)(\theta, z, k):=\sum_{n=0}^{N} \phi_{n}(z) \sum_{m=0}^{\infty} \varepsilon_{m} \int_{\partial \Omega}\left(\frac{\partial}{\partial v}+\lambda\right) \phi_{n}(\zeta) J_{m}\left(k a_{n} r^{\prime}\right) \cos m\left(\theta-\theta^{\prime}\right) d \sigma
$$

We know that

$$
\begin{gathered}
\psi_{n m}^{1}:=\left(\frac{\partial}{\partial v}+\lambda\right)\left[\phi_{n}(\zeta) J_{m}\left(k a_{n} r^{\prime}\right) \cos m \theta\right] \\
\psi_{n m}^{2}:=\left(\frac{\partial}{\partial v}+\lambda\right)\left[\phi_{n}(\zeta) J_{m}\left(k a_{n} r^{\prime}\right) \sin m \theta\right], \\
(r, \theta, z) \in \partial \Omega, n, m=0,1, \ldots, \infty
\end{gathered}
$$

are a complete system in $L^{2}(\partial \Omega),[6]$. Let

$$
W_{N}(\partial \Omega):=\overline{\operatorname{span}\left\{\psi_{n m}^{1}, \psi_{n m}^{2} ; n=0,1, \ldots, N ; m=0,1, \ldots, \infty\right\}}
$$

and $W_{N}^{\frac{1}{N}}(\partial \Omega)$ be the orthogonal space to $W_{N}(\partial \Omega)$ in $L^{2}(\partial \Omega)$ under the usual $L^{2}(\partial \Omega)$ inner product, then $N(F)=W_{N}^{\perp}(\partial \Omega)$, here $N(F)$ is the null space of the far-field pattern operator $F$. Hence, if $g \in W_{N}^{\perp}(\partial \Omega)$, then from (2.6)

$$
u^{s}(\mathbf{x}, z)=O\left(\frac{1}{r^{3 / 2}}\right)
$$

i.e. the propagating far-field pattern of $\boldsymbol{u}^{s}$ is identical to zero.

Now we want to formulate a mapping from incoming waves to far-field pattern. At this stage, we think of the object $\Omega$ as known and fixed. Let

$$
A\left(k, R_{b}^{3}\right):=\left\{u ; u(\mathbf{x}, z)=\sum_{n=0}^{\infty} \sum_{m=-\infty}^{\infty} \alpha_{n m} \phi_{n}(z) J_{m}\left(k a_{n} r\right) e^{i m \theta}, \quad(\mathbf{x}, z) \in \mathbf{R}_{b}^{3}\right\}
$$

for any $u^{i} \in A\left(k, \mathbf{R}_{b}^{3}\right)$, denote $u_{b}^{i}=\left.u^{i}\right|_{\partial \Omega}$ which is a continuous function on $\partial \Omega$. Since $(I+K+\lambda S)$ is invertible for any $k>0$, we can express $g \in L^{2}(\partial \Omega)$ as

$$
g(\mathbf{x}, z)=-2(I+K+\lambda S)^{-1} u_{b}^{i}, \quad(\mathbf{x}, z) \in \partial \Omega
$$


Combining (2.7) and (2.10), we define a mapping $\hat{F}_{a n} A\left(k, \mathbf{R}_{b}^{3}\right) \rightarrow V^{N}$ by

$$
\hat{F}_{\partial \Omega} u^{i}:=F \circ(I+K+\lambda S)^{-1}\left(-2 u_{b}^{i}\right) .
$$

Let

$$
\begin{aligned}
& A(N, \partial \Omega):=\left\{u^{i} \in A\left(k, \mathbf{R}_{b}^{3}\right),(I+K+\lambda S)^{-1} u_{b}^{i} \in W_{N}(\partial \Omega)\right\} \\
& A_{1}(N, \partial \Omega):=\left\{u^{i} \in A\left(k, \mathbf{R}_{b}^{3}\right),(I+K+\lambda S)^{-1} u_{b}^{i} \in W_{N}^{1}(\partial \Omega)\right\}
\end{aligned}
$$

then we can see from (2.9) that $N\left(\hat{F}_{\partial \Omega}\right)=A_{1}(N, \partial \Omega)$.

Definition 1. Let $u_{1}^{i}, u_{2}^{i} \in A\left(k, \mathbf{R}_{b}^{3}\right)$ be two incoming waves, we say that $u_{1}^{i}$ is equivalent to $u_{2}^{i}$ if $u_{1}^{i}-u_{2}^{i} \in A_{1}(N, \partial \Omega)$, which is denoted by $u_{1}^{i} \sim u_{2}^{i}$.

Let $\left\{u^{i}\right\}$ be the equivalent class under this equivalent relation $\sim$, then for any given far-field pattern $f \in \mathbf{R}\left(\hat{F}_{\partial \Omega}\right)$, the range of $\hat{F}_{\partial \Omega}$, there exists an equivalent class $\left\{u^{i}\right\}$, such that for any element in the class,

$$
\hat{F}_{\partial \mathbf{\Omega}} u^{i}=f
$$

We call $\left\{u^{i}\right\}$ an equivalent class solution.

Define

$$
\left\|u^{i}\right\|_{\partial \Omega}^{2}:=\int_{\partial \Omega}\left|(I+K+\lambda S)^{-1} u_{b}^{i}\right|^{2} d \sigma
$$

then we call $u^{i} \in A\left(k, \mathbf{R}_{b}{ }^{3}\right)$ a minimal norm solution of integral equation (2.15) if

$$
\hat{F}_{\partial \boldsymbol{\Omega}} u^{i}=f
$$

such that

$$
\left\|u^{i}\right\|_{\partial \Omega}=\inf _{u^{i} \in\left[u^{i}\right\}}\left\|u^{i}\right\|_{\partial \Omega}
$$

Theorem 2.1. If $u^{i} \in A(N, \partial \Omega)$, such that $\hat{F}_{\partial \Omega} u^{i}=0$, then

$$
u^{i}=0, \quad \text { on } \partial \Omega
$$

Proof. We have $u^{i} \in A(N, \partial \Omega)$, so $g:=(I+K+\lambda S)^{-1} u_{b}^{i} \in W_{N}(\partial \Omega)$. We can represent $\hat{F}_{\partial \Omega} u^{i}$ as

$$
\left(\hat{F}_{\partial \Omega} u^{i}\right)(\theta, z)=F g=\sum_{n=0}^{N} \sum_{m=0}^{\infty} \varepsilon_{m} \phi_{n}(z)
$$




$$
\begin{gathered}
\int_{\partial \Omega}\left(\frac{\partial}{\partial v}+\lambda\right) \phi_{n}(\zeta) J_{m}\left(k a_{n} r^{\prime}\right) \cos m\left(\theta-\theta^{\prime}\right) g(\xi, \zeta) d \sigma=0 \\
(\theta, z) \in[0,2 \pi] \times[0, h]
\end{gathered}
$$

It follows that

$$
\int_{\partial \Omega}\left(\frac{\partial}{\partial v}+\lambda\right) \psi_{m n}^{i} g d \sigma=0, \quad i=1,2 ; \quad n=0,1, \ldots, N ; \quad m=0,1, \ldots, \infty
$$

Hence, $g \in W_{N}^{\frac{1}{N}}(\partial \Omega)$, and $g=0$ on $\partial \Omega$. Consequently, $u_{b}^{i}=(I+K+\lambda S) g=0$ on $\partial \Omega$.

Corollary. Let $\left\{u^{i}\right\}$ be an equivalent class solution of (2.15), then there is a unique $u_{0}^{i} \in A(N, \partial \Omega)$ such that any element of $\left\{u^{i}\right\}$ can be written as

$$
u^{i}=u_{0}^{i}+u_{1}^{i}
$$

where $u_{1}^{i} \in A_{1}(k, \partial \Omega)$.

Since

$$
\begin{aligned}
\left\|u^{i}\right\|_{\partial \Omega}^{2} & =\left\|u_{0}^{i}+u_{1}^{i}\right\|_{\partial \Omega}^{2} \\
& =\int_{\partial \Omega}\left|(I+K+\lambda S)^{-1}\left(u_{0}^{i}+u_{1}^{i}\right)\right|^{2} d \sigma \\
& =\int_{\partial \Omega}\left|(I+K+\lambda S)^{-1} u_{0}^{i}\right|^{2} d \sigma+4 \int_{\partial \Omega}\left|(I+K+\lambda S)^{-1} u_{1}^{i}\right|^{2} d \sigma \\
& =\left\|u_{0}^{i}\right\|_{\partial \Omega}^{2}+\left\|u_{1}^{i}\right\|_{\partial \Omega}^{2},
\end{aligned}
$$

$\left\|u^{i}\right\|_{\partial \Omega} \geqq\left\|u_{o}^{i}\right\|_{\partial \Omega}$ for any element of $\left\{u^{i}\right\}$, from which we can conclude:

Theorem 2.2. Let $\left\{u^{i}\right\}$ be the equivalent class solution of (2.15), which has a unique decomposite expression

$$
u^{i}=u_{0}^{i}+u_{1}^{i}, \quad u_{0}^{i} \in A(N, \partial \Omega), \quad u_{1}^{i} \in A_{1}(N, \partial \Omega)
$$

then $u_{0}^{i}$ is the minimal norm solution of (2.14).

Theorem 2.3. If $u^{i} \in A(N, \partial \Omega)$ such that the corresponding propagating far-field pattern $f(\theta, z)=0$, then the corresponding scattered wave $u^{5}=0$ in $\mathbf{R}_{b}^{3} \backslash \Omega$.

Proof. Let $u^{i} \in A(N, \partial \Omega)$, such that 


$$
\hat{F}_{\partial \Omega} u^{i}=f=0 .
$$

By Theorem 2.1, $u^{i}=0$ on $\partial \Omega$. Hence $u^{s}=-u^{i}=0$, on $\partial \Omega$. The uniqueness theorem of direct scattering problem (cf. [4]) follows

$$
\boldsymbol{u}^{s}=0, \quad \text { in } \mathbf{R}_{b} \backslash \mathbf{\Omega} .
$$

\section{An alternative injective theorem}

As pointed out in the last section, $\hat{F}_{\partial \Omega}: A\left(k, \mathbf{R}_{b}^{3}\right) \rightarrow V^{N}$ is not an injection; however, we can restrict $\hat{F}_{\partial \Omega}$ on a linear subspace related to $\partial \Omega$ so that $\hat{F}_{\partial \boldsymbol{\Omega}}$ is an injection in the linear subspace. One possible choice for this purpose is to take $\overline{A(N, \partial \Omega)}$ as the domain of $\hat{F}_{\partial \Omega}$. However, in order to formulate the inverse problem in terms of single layer potentials, which has proved efficient in the $\mathbf{R}^{3}$ case in [10], we need to introduce a different restriction on $\hat{F}_{\text {an }}$.

We first prove the following lemma.

Lemma 3.1. Let $D$ be a bounded convex region in $\mathbf{R}_{b}^{3}$, such that $k>0$ is not a Dirichlet eigenvalue of $D$, then

$$
\begin{aligned}
& \mu_{m n}^{(1)}:=\phi_{n}(z) J_{m}\left(k a_{n} r\right) \cos m \theta, \\
& \mu_{m n}^{(2)}:=\phi_{n}(z) J_{m}\left(k a_{n} r\right) \sin m \theta,
\end{aligned}
$$

are complete in $L^{2}(\partial D)$.

Proof. It suffices to show that if $g \in L^{2}(\partial D)$, such that

$$
\begin{gathered}
\int_{\partial \Omega} g(r, z, \theta)\left[\phi_{n}(z) J_{m}\left(k a_{n} r\right) \cos (m \theta)\right] d \sigma=0, \\
\int_{\partial \Omega} g(r, z, \theta)\left[\phi_{n}(z) J_{m}\left(k a_{n} r\right) \sin (m \theta)\right] d \sigma=0,
\end{gathered}
$$

for $m, n=0,1, \ldots, \infty$, then $g$ is identically zero on $\partial D$.

Let

$$
u(\mathbf{x}, z):=\int_{\partial \Omega} G(z, \zeta,|\mathbf{x}-\xi|) g\left(r^{\prime}, \zeta^{\prime}, \theta^{\prime}\right) d \sigma
$$

then $u \equiv 0$ for $|\mathbf{x}|$ sufficiently large. But $u$ is a solution to the Helmholtz equation, so $u$ $=0$ in $\mathbf{R}_{b}^{3} \backslash D$ by the analyticity of $u$. Moreover,

$$
u_{+}-u_{-}=2 g, \text { on } \partial D
$$


and

$$
\left(\frac{\partial u}{\partial v}\right)_{+}-\left(\frac{\partial u}{\partial v}\right)_{-}=-2 \lambda g, \text { on } \partial D
$$

Since $u_{+}=0$, we know $u_{-}=0$ on $\partial D$. By assumption, $k$ is not a Dirichlet eigenvalue of $D$, so $u \equiv 0$ in $D$. It follows that

$$
g=-\frac{1}{2}\left(\frac{\partial u_{+}}{\partial v}-\frac{\partial u_{-}}{\partial v}\right)=0, \quad \text { on } \partial D
$$

Now we can represent the solution to the exterior Dirichlet problem in the form of an acoustic single-layer potential

$$
u^{s}(\mathbf{x}, z):=\int_{\partial D} G(z, \zeta,|\mathbf{x}-\xi|) g\left(r^{\prime}, \zeta^{\prime}, \theta^{\prime}\right) d \sigma, \quad(\mathbf{x}, z) \in \mathbf{R}_{b}^{3} \backslash \Omega
$$

where $D$ is an auxiliary region contained in $\Omega$.

The potential (3.6) solves the exterior Dirichlet problem provided that the density $\phi$ is a solution of the integral equation of the first kind

$$
\int_{\partial D} G(z, \zeta,|\mathbf{x}-\xi|) \phi(\xi, \zeta) d \sigma_{\xi}=-u^{i}(\mathbf{x}, z), \quad(\mathbf{x}, z) \in \partial \Omega
$$

We introduce an integral mapping $T: L^{2}(\partial D) \rightarrow L^{2}(\partial \Omega)$ by

$$
(T \phi)(\mathbf{x}, z):=\int_{\partial D} G(z, \zeta,|\mathbf{x}-\xi|) \phi(\xi, \zeta) d \sigma_{\xi}, \quad(\mathbf{x}, z) \in \partial \Omega
$$

and write (3.8) as

$$
T \phi=-u^{i}
$$

Since the boundary $\partial \Omega$ and the auxiliary surface $\partial D$ are disjoint, the integral operator $T$ has a smooth kernel and therefore it is compact and cannot have a bounded inverse. Hence, the integral equation (3.10) is ill-posed.

However, it is not our purpose to solve the direct problem by solving (3.10). We are concerned with finding a linear subspace of $A\left(k, \mathbf{R}_{b}^{3}\right)$ so that the restriction of the far-field pattern operator $F$ to this subspace is injective.

Here we remark that, similar to the case discussed in [10], equation (3.10) can have a solution only for those incoming waves $u^{i}$ for which the scattered wave $u^{s}$ can be analytically extended into the exterior of $\partial D$. Some discussion related to this question may be found in [11] and [13]. However, for an arbitrary region this is still an open problem. 
Suppose for a region $\Omega$ and an incoming wave $u^{i}$ the equation (3.10) has a solution $\phi$, then we can write the far-field pattern operator $F_{\partial \Omega}: A\left(k, \mathbf{R}_{b}^{3}\right) \rightarrow V^{N}$ in the form of

$$
F_{\partial \mathbf{\Omega}} u^{i}=F_{1} \phi:=\sum_{n=0}^{N} \sum_{m=0}^{\infty} \varepsilon_{m} \phi_{n}(z) \int_{\partial \Omega} \phi_{n}(\zeta) J_{m}\left(k a_{n} r^{\prime}\right) \cos m\left(\theta-\theta^{\prime}\right) \phi(\xi, \zeta) d \sigma
$$

where $\phi \in L^{2}(\partial D)$ is a solution of $(3.10)$. Let

$$
\begin{gathered}
U_{N}:=\overline{\operatorname{span}\left\{\mu_{m n}^{(1)}, \mu_{m n}^{(2)} ; n=0,1, \ldots, N ; m=0,1, \ldots, \infty\right\}} \\
\mathbf{U}_{N}^{\perp}:=\left\{u \in L^{2}(\partial D) ; \int_{\partial D} u \bar{v} d \sigma=0 \text { for any } v \in U_{N}\right\} \\
T U_{N}:=\left\{u \in L^{2}(\partial D) ; u=T \phi ; \text { for some } \phi \in U_{N}\right\} \\
T U_{N}^{\frac{1}{N}}:=\left\{u \in L^{2}(\partial D) ; u=T \phi ; \text { for some } \phi \in U_{N}^{\perp}\right\} \\
B(N, \partial \Omega):=\left\{u \in A\left(k, \mathbf{R}_{b}^{3}\right) ;\left.u\right|_{\partial \Omega} \in \overline{T U_{N}}\right\} \\
B(N, \partial \Omega):=\left\{u \in A\left(k, \mathbf{R}_{b}^{3}\right) ;\left.u\right|_{\partial \Omega} \in T U_{N}^{\perp}\right\}
\end{gathered}
$$

Theorem 3.2.

$$
N\left(F_{\partial \Omega}\right) \supset B_{1}(N, \partial \Omega)
$$

Proof. If $u^{i} \in B_{1}(N, \partial \Omega)$, then there is a function $\phi \in U_{N}^{\perp}$ such that

$$
T \phi=\left.u^{i}\right|_{\partial \Omega}
$$

Hence,

$$
F_{\partial \Omega} u^{i}=\sum_{n=0}^{N} \sum_{m=0}^{\infty} \varepsilon_{m} \phi_{n}(z) \int_{\partial D} \phi_{n}(\zeta) J_{m}\left(k a_{n} r^{\prime}\right) \cos m\left(\theta-\theta^{\prime}\right) \phi(\xi, \zeta) d \sigma=0
$$

due to the fact that

$$
\begin{gathered}
\int_{\partial D} \phi(\xi, \zeta)\left[\phi_{n}(\zeta) J_{m}\left(k a_{n} r^{\prime}\right) \cos \left(m \theta^{\prime}\right)\right] d \sigma=0, \\
\int_{\partial D} \phi(\xi, \zeta)\left[\phi_{n}(\zeta) J_{m}\left(k a_{n} r^{\prime}\right) \sin \left(m \theta^{\prime}\right)\right] d \sigma=0, \\
\text { for } m=0,1, \ldots, \infty, n=0,1, \ldots, N .
\end{gathered}
$$


Theorem 3.3. Suppose $u^{i} \in A\left(k, \mathbf{R}_{b}^{3}\right)$ and equation (3.10) has a solution in $L^{2}(\partial D)$. If $F_{\text {on }} u^{i}=0$ then $u^{i} \in B_{1}(N, \partial \Omega)$.

Proof. For $u^{i} \in A\left(k, \mathbf{R}_{b}^{3}\right)$, let $\phi \in L^{2}(\partial D)$ be a solution of (3.10), then the scattered wave $u^{s}$ can be written as

$$
u^{s}(\mathbf{x}, z):=\int_{\partial D} G(z, \zeta,|\mathbf{x}-\xi|) \phi(\xi, \zeta) d \sigma
$$

For $r=|\mathbf{x}| \rightarrow \infty$, we have

$$
\begin{gathered}
F_{\partial \Omega} u^{i}=\sum_{n=0}^{N} \phi_{n}(z) \sum_{m=0}^{\infty} \varepsilon_{m}\left\{\left[\int_{\partial D} \phi(\xi, \zeta) \phi_{n}(\zeta) J_{m}\left(k a_{n} r^{\prime}\right) \cos \left(m \theta^{\prime}\right) d \sigma\right] \cos m \theta\right. \\
+\left[\int_{\partial D} \phi(\xi, \zeta)\left[\phi_{n}(\zeta) J_{m}\left(k a_{n} r^{\prime}\right) \sin \left(m \theta^{\prime}\right) d \sigma\right] \sin m \theta\right\}=0 \\
0 \leqq z \leqq h, 0 \leqq \theta \leqq 2 \pi .
\end{gathered}
$$

It follows that

$$
\begin{gathered}
\int_{\partial D} \phi(\xi, \zeta)\left[\phi_{n}(\zeta) J_{m}\left(k a_{n} r^{\prime}\right) \cos \left(m \theta^{\prime}\right)\right] d \sigma=0 \\
\int_{\partial D} \phi(\xi, \zeta)\left[\phi_{n}(\zeta) J_{m}\left(k a_{n} r^{\prime}\right) \sin \left(m \theta^{\prime}\right)\right] d \sigma=0 \\
\quad \text { for } m=0,1, \ldots, \infty, n=0,1, \ldots, N
\end{gathered}
$$

Hence $\phi \in U_{N}^{\perp}$ and $\left.u^{i}\right|_{\partial \Omega}=-T \phi \in T U_{N}^{\perp}$.

Corollary. Suppose $u^{i} \in A\left(k, \mathbf{R}_{b}^{3}\right)$ and equation (3.10) has a solution in $B(N, \partial D)$. If $F_{\partial \Omega} u^{i}=0$, then $u^{i}=0$ and

$$
u^{3}=\mathbf{0} \quad \text { in } \mathbf{R}_{b}^{3} \backslash \Omega
$$

4. The inverse problem and its approximation solutions

In view of Section 3 , if $u^{i}$ is an incoming wave which admits a solution to equation (3.10), i.e.

$$
T \phi=-u^{i}, \quad \phi \in L^{2}(\partial D)
$$


then we can introduce a far-field operator $F_{1}: L^{2}(\partial D) \rightarrow V^{N}$ as:

$$
\begin{gathered}
F_{1} \phi:=\sum_{n=0}^{N} \sum_{m=0}^{\infty} \varepsilon_{m} \phi_{n}(z) \int_{\partial D} \phi_{n}(\zeta) J_{m}\left(k a_{n} r^{\prime}\right) \cos m\left(\theta-\theta^{\prime}\right) \phi(\zeta, \zeta) d \sigma, \\
0 \leqq z \leqq h, 0 \leqq \theta \leqq 2 \pi .
\end{gathered}
$$

For a given far-field pattern, it leads to an integral equation of the first kind, namely

$$
F_{1} \phi=f, \text { on } \Gamma \text {, }
$$

where $\Gamma:=\{(1, \theta, z) ; 0 \leqq \theta \leqq 2 \pi, 0 \leqq z \leqq h\}$.

We know that $F_{1}$ is an injection if $k$ is not a Dirichlet eigenvalue of $D$ and the domain of $F_{1}, D\left(F_{1}\right)$, is $U_{N}$. However, we cannot expect in general that a solution to (4.3) exists.

One of the basic techniques to treat ill-posed integral equations of the first kind is the classical Tikhonov functional

$$
\left\|F \phi_{\alpha}-f\right\|_{L^{2}(\Gamma)}^{2}+\alpha\left\|\phi_{\alpha}\right\|_{L^{2}(\partial D)}^{2} .
$$

After we have determined $\phi_{a}$ and the corresponding approximation $u_{a}^{s}$ for the scattered wave $u^{s}$, we look for the unknown surface $\partial \Omega$ as the location of the zeros of $u_{\alpha}^{s}+u^{i}$. As suggested in the whole space case (cf. [10,2]), we make an a priori assumption on the unknown surfaces that if $U$ is the set of all possible surfaces, the elements of $U$ can be described by

$$
\Lambda:=\left\{\left(0,0, z_{0}\right)+r(\mathbf{x}) \mathbf{x} ; \mathbf{x} \in B\right\},
$$

where $B$ is the unit sphere and $0<z_{0}<h$ is a known constant, $r(\mathbf{x})$ belongs to a compact subset

$$
V:=\left\{r \in C^{1 \cdot \beta}(B) ; 0 \leqq r_{1}(\mathbf{x}) \leqq r(\mathbf{x}) \leqq r_{2}(\mathbf{x})\right\} .
$$

As usual, $C^{1, \beta}(B), 0<\beta \leqq 1$, denotes the space of uniformly Holder continuously differentiable functions on the unit sphere furnished with the appropriate Holder norm. The functions $r_{1}(\mathbf{x})$ and $r_{2}(\mathbf{x})$ in the definition of $V$ represent the $a$ priori information.

If $\partial D$ is contained in the interior of the surface represented by $r(x) x+\left(0,0, z_{0}\right)$, (for simplification, we sometimes just say by $r(\mathbf{x}))$, we locate $\partial \Omega$ by minimizing

$$
\int_{\Lambda}\left|u_{\alpha}^{s}+u^{i}\right|^{2} d \sigma
$$

over all surfaces $\Lambda$ in $U$; or, similar to [10], neglecting the Jacobian of $r(x)$, by minimizing 


$$
\int_{B}\left|\left(u_{\alpha}^{s}+u^{i}\right) \circ r\right|^{2} d \sigma
$$

over all functions $r \in V$.

Combining (4.4) and (4.5), we can formulate the inverse problem as minimizing the functional:

$$
\mu(\phi, r ; f, \alpha):=\|F \phi-f\|_{L^{2}(\Gamma)}^{2}+\alpha\|\phi\|_{L^{2}(\partial D)}^{2}+\left\|\left(T \phi+u^{i}\right) \circ r\right\|_{L^{2}(B)}^{2} .
$$

Here we use $T$ to denote the single-layer acoustic potential

$$
(T \phi)(\mathbf{x}, z):=\int_{\partial D} G(z, \zeta,|\mathbf{x}-\xi|) \phi d \sigma ;(\mathbf{x}, z) \in \mathbf{R}_{b}^{3} \backslash \partial D .
$$

That is, we seek $\phi^{*} \in U_{N}$ and $r^{*} \in V$ such that

$$
\mu\left(\phi^{*}, r^{*} ; f, \alpha\right)=M(f, \alpha):=\inf \left\{\mu(\phi, r ; f, \alpha) ; \phi \in U_{N}, r \in V\right\}
$$

Now we establish existence of a solution to this nonlinear optimization problem and investigate its convergent property as $\alpha \rightarrow 0$.

Theorem 4.1. The optimization formulation of the inverse scattering problem has a solution.

Proof. Let $\left(\phi_{n}, r_{n}\right) \in U_{N} \times V$ be a minimizing sequence. This means that

$$
\lim _{n \rightarrow \infty} \mu\left(\phi_{n}, r_{n} ; f, \alpha\right)=M(f, \alpha)
$$

Since $V$ is compact, we may assume that $r_{n} \rightarrow r \in U$, as $n \rightarrow \infty$.

In view of

$$
\alpha_{n}\left\|\phi_{n}\right\|_{L^{2}(\partial D)}^{2} \leqq \mu\left(\phi_{n}, r_{n} ; f, \alpha\right) \rightarrow M(f, \alpha), \quad n \rightarrow \infty
$$

and $\alpha>0$, we know that the sequence $\left\{\phi_{n}\right\}$ is bounded. Hence, we may conclude that $\left\{\phi_{n}\right\}$ converges weakly to some $\phi \in U_{N}$ as $n \rightarrow \infty$. From the fact that $F$ and $T$ are compact operators it follows that

$$
F \phi_{n} \rightarrow F \phi, \quad n \rightarrow \infty
$$

and

$$
\left(T \phi_{n}\right) \circ r_{n} \rightarrow(T \phi) \circ r, \quad n \rightarrow \infty
$$

But then from (4.7) we know 


$$
\left\|\phi_{n}\right\|_{L^{2}(\partial D)}^{2} \rightarrow\|\phi\|_{L^{2}(\partial D)}^{2}, \quad n \rightarrow \infty
$$

This, together with the weak convergence, implies that

$$
\left\|\phi_{n}-\phi\right\|_{L^{2}(\partial D)} \rightarrow 0, \quad n \rightarrow \infty
$$

and $\phi \in U_{N}$ due to the fact that $U_{N}$ is a closed set. Hence

$$
\mu(\phi, r ; f, \alpha)=\lim _{n \rightarrow \infty} \mu\left(\phi_{n}, r_{n} ; f, \alpha\right)=M(f, \alpha) \text {. }
$$

This completes the proof.

Theorem 4.2. Let $u^{i} \in B(N, \partial \Omega)$ and $f_{0}$ be the corresponding far-field pattern of $a$ domain $\partial \Omega$ which is described by some $r \in V$, then

$$
\lim _{\alpha \rightarrow 0} M\left(f_{0}, \alpha\right)=0
$$

Proof. Let $\varepsilon>0$ be arbitrary, then there exists $\phi \in U_{N}$ such that

$$
\left\|\left(T \phi+u^{i}\right) \circ r\right\|_{L^{2}(B)}<\varepsilon .
$$

Since the far-field pattern of the scattered wave depends continuously on the boundary data of $u^{s}$, we can find a constant depending on $\partial \Omega, C=C(\partial \Omega)$, such that

$$
\left\|F_{1} \phi-f_{0}\right\|_{L^{2}(\Gamma)} \leqq C\left\|\left(T \phi-u^{s}\right) \circ r\right\|_{L^{2}(B)} .
$$

In view of $u^{i}+u^{s}=0$ on $\partial \Omega$, we have

$$
\begin{aligned}
\mu\left(\phi, r ; f_{0}, \alpha\right) & \leqq(1+C)\left\|\left(T \phi+u^{i}\right) \circ r\right\|_{L^{2}(B)}+\alpha\|\phi\|_{L^{2}(\partial D)} \\
& \leqq(1+C) \varepsilon+\alpha\|\phi\| \rightarrow(1+C) \varepsilon, \alpha \rightarrow .
\end{aligned}
$$

From the above we have the following result.

Theorem 4.3. Let $u^{i} \in B(N, \partial \Omega)$ be an incoming wave such that $\left.u^{i}\right|_{\partial \Omega} \in T U_{N}$ and $f$ be the corresponding far-field pattern of a domain $\Omega$ such that $\partial \Omega$ is described by a null sequence and let $\left(\phi_{n}, r_{n}\right)$ be a solution to the minimization problem with regularization parameter $\alpha_{n}$. Then there exists a convergent subsequence of the sequence $\left\{r_{n}\right\}$. There is only a finite number of limit points and every limit point represents a surface on which the total field $u^{s}+u^{i}$ vanishes.

Proof. From the compactness of $V$, there exists a convergent subsequence of $\left\{r_{n}\right\}$ which converges to, say, $r^{*}$. Without loss of generality, we may assume that $r_{n} \rightarrow r^{*}$, as 
$n \rightarrow \infty$. Let $u^{*}$ denote the unique solution to the direct scattering problem for the object with boundary $\Lambda^{*}$ described by $r^{*}$, then

$$
\left(u^{*}+u^{i}\right) \circ r^{*}=0, \quad \text { on } B .
$$

Here we can think of that $u_{n}$ as the solution to an exterior Dirichlet problem with boundary values $\left.T \phi_{n}\right|_{\Lambda_{n}}$ on the boundary $\Lambda_{n}$ described by $r_{n}$.

Similar to the proof of Theorem 2.2 in [2] (also cf. [10]), we can show the following lemma.

Lemma. Let $\left\{r^{*}\right\}, r^{*}$ be surfaces in $\mathbf{R}_{b}^{3}, r_{n} \rightarrow r^{*}$ as $n \rightarrow \infty$. Let $u^{i}$ be an incoming wave, $\left\{u_{n}\right\}$ and $u^{*}$ be scattered waves satisfying

$$
\begin{gathered}
\left(u^{*}+u^{i}\right) \circ r^{*}=0, \quad \text { on } B ; \\
\left\|\left(u_{n}+u^{i}\right) \circ r_{n}\right\|_{L^{2}(B)} \rightarrow 0, \quad \text { as } n \rightarrow \infty ;
\end{gathered}
$$

then for any closed set $G$ in $\mathbf{R}_{b}{ }^{3} \backslash D$,

$$
\left\|u_{n}-u^{*}\right\|_{\infty, G} \rightarrow 0, \quad n \rightarrow \infty .
$$

where $D$ is contained in the interior region of $r^{*}$ and $\|\cdot\|_{\infty, G}$ is the maximum norm over $G$.

From the lemma we know the far-field patterns $F_{1} \phi_{n}$ of $u_{n}$ converge uniformly to the far-field pattern $f^{*}$ of $u^{*}$. Moreover, by Theorem 4.2 ,

$$
\left\|F_{1} \phi_{n}-f\right\|_{L^{2}(\Gamma)} \rightarrow 0, \text { as } n \rightarrow \infty .
$$

Therefore, we can conclude that the far-field patterns coincide

$$
f=f^{*} \text {. }
$$

Recall that $f$ is the far-field pattern with respect to an incoming wave $u^{i} \in B(N, \partial \Omega)$ such that $T \phi=-u^{i}$ admits a solution $\phi_{0} \in U_{N}$. Therefore, we can represent the scattered wave as:

$$
u^{s}(\mathbf{x}, z)=\left(T \phi_{0}\right)(\mathbf{x}, z), \quad(\mathbf{x}, z) \in \mathbf{R}_{b}^{3} \backslash \Omega .
$$

Since $f=F_{1} \phi_{0}$,

$$
\left\|F_{1}\left(\phi_{n}-\phi_{0}\right)\right\|_{L^{2}(\Gamma)}=\left\|F_{1} \phi_{n}-f\right\|_{L^{2}(\Gamma) \rightarrow 0,} \text { as } n \rightarrow \infty
$$

Now (4.2) implies that

$$
\int_{\partial D}\left[\phi_{n}-\phi_{0}\right]\left[\phi_{n}(\zeta) J_{m}\left(k a_{n} r^{\prime}\right) \cos \left(m \theta^{\prime}\right)\right] d \sigma \rightarrow 0
$$




$$
\int_{\partial D}\left[\phi_{n}-\phi_{0}\right]\left[\phi_{n}(\zeta) J_{m}\left(k a_{n} r^{\prime}\right) \sin \left(m \theta^{\prime}\right)\right] d \sigma \rightarrow 0
$$

when $n \rightarrow \infty$. It follows immediately that

$$
\left\|T \phi_{n}-u^{s}\right\|_{\infty, G}=\left\|T\left(\phi_{n}-\phi_{0}\right)\right\|_{\infty, G} \rightarrow 0, \text { as } n \rightarrow \infty
$$

Consequently,

$$
\begin{aligned}
& \left\|u^{s}-u^{*}\right\|_{\infty, G} \leqq\left\|u^{s}-T \phi_{n}\right\|_{\infty, G}+\left\|T \phi_{n}-u^{*}\right\|_{\infty, G} \\
& =\left\|T\left(\phi_{n}-\phi_{0}\right)\right\|_{\infty, G}+\left\|u_{n}-u^{*}\right\|_{\infty, G} \rightarrow 0, \quad n \rightarrow \infty,
\end{aligned}
$$

due to (4.14) and (4.16), where $G$ is any closed set in $\mathbf{R}_{b}^{3} \backslash D$. In view of (4.17) and that $u^{*}+u^{i}=0$ on $\Lambda$ and $\Lambda^{*} \subset \mathbf{R}_{b}^{3} \backslash D$, we can conclude that

$$
u^{s}+u^{i}=0, \quad \text { on } \Lambda^{*}
$$

If there existed an infinite number of different limit points, then by the compactness of $V$ we could find a convergent sequence of these limit points. Thus it would follow that there was an arbitrarily small region for which $u^{s}+u^{i}$ is an eigenfunction for the Laplacean. This is impossible; hence the number of limit points is finite.

\section{REFERENCES}

1. D. Ahluwalia and J. Keller, Exact and asymptotic representations of the sound field in a stratified ocean, in Wave Propagation and Underwater Acoustics (Lecture Notes in Physics 70, Springer, Berlin 1977).

2. T. S. Angell, D. Colton and A. KIRSCH, The three dimensional inverse scattering problem for acoustic waves, J. Differential Equations 46 (1982), 46-58.

3. T. S. Angell, R. E. Kleiman and G. F. Roach, An inverse transmission problem for the Helmholtz equation, Inverse Problems 3 (1987), 149-180.

4. D. Colton, The inverse scattering problem for time-harmonic acoustic waves, SIAM Rev. 26 (1984), 323-350.

5. D. Colton, and P. Monk, A novel method of solving the inverse scattering problem for time-harmonic acoustic waves in the resonance region, SIAM J. Appl. Math. 45 (1985), 1039-1053.

6. D. Colton and P. Monk, A novel method of solving the inverse scattering problem for time-harmonic acoustic waves in the resonance region: II, SIAM J. Appl. Math. 46 (1986), 506-523.

7. D. Colton and R. Kress, Integral Equation Methods in Scatttering Theory (John Wiley, New York, 1983).

8. R. P. Gilbert and Yongzhı Xu, Starting fields and far fields in ocean acoustics, Wave Motion 11 (1989), 507-524.

9. R. G. Gilbert and Yongzhı Xu, Dense sets and the projection theorem for acoustic harmonic waves in homogeneous finite depth ocean, Math. Methods Appl. Sci. 12 (1989), 69-76. 
10. A. KIRSCH, and R. Kress, An optimization method in inverse acoustic scattering, in Boundary Elements, Vol. 3. Fluid Flow and Potential Applications (1988), to appear.

11. R. F. Millar, The Rayleigh hypothesis and a related least-squares solution to scattering problems for periodic surfaces and other scatterers, Radio Science 8 (1973), 785-796.

12. B. D. Sleeman, The inverse problem of acoustic scattering, IMA J. Appl. Math. 29 (1982), $13-142$.

13. P. M. VAN DEN BERG and J. T. FoKkema, The Rayleigh hypothesis in the theory of diffraction by a cylindrical obstacle, IEEE Trans. Antennas and Propagation AP-27 (1979), 577-583.

14. YongzHI $\mathrm{XU}$, The propagating solution and far field patterns for acoustic harmonic waves in a finite depth ocean, Appl. Anal. 35 (1990), 129-151.

InSTITUTE for Mathematics AND its APPLiCations

UNIVERSITY OF MiNNESOTA

MiNNEAPOLIS, MN 55455

USA 